\title{
ACCRETION ONTO RELATIVISTIC OBJECTS
}

\author{
M. J. REES* \\ Astronomy Centre, University of Sussex, Falmer, Brighton BN1 9QH, England
}

\begin{abstract}
The physics of spherically symmetrical accretion onto a compact object is briefly reviewed. Neither neutron stars nor stellar-mass black holes are likely to be readily detectable if they are isolated and accreting from the interstellar medium. Supermassive black holes in intergalactic space may however be detectable. The effects of accretion onto compact objects in binary systems are then discussed, with reference to the phenomena observed in variable $\mathrm{X}$-ray sources.
\end{abstract}

\section{Introduction}

Early studies of accretion (Bondi, 1952; Mestel, 1954 and earlier references cited therein) were motivated by the hope that infalling interstellar matter might cause a substantial increase in the luminosity of ordinary stars. The effects turned out to be generally insignificant, for two reasons: (i) the gravitational potential $G M / r$ at the surface of an ordinary star is only $\sim 10^{-6} c^{2}$, implying an energy release per unit mass which is very small compared to that available from nuclear reactions; and (ii) the low density of surrounding matter means that the accretion rate onto an isolated star is low.

Accretion onto neutron stars or black holes $\left(G M / r \gtrsim 0.1 c^{2}\right)$ is obviously likely to be more significant, because each gram of infalling matter may yield $\gtrsim 10^{20} \mathrm{erg}$ of electromagnetic energy; and a search for the radiation thus emitted is surely one of the most promising ways of detecting such objects. The recent evidence for compact objects in close binary systems has been a stimulus for much further work on this subject, because one here expects more spectacular observable effects, since there should be not only a large energy release per gram, but also a high accretion rate owing to the presence of a close companion star.

In this Paper I shall first briefly discuss accretion onto isolated compact objects, and then consider some theoretical implications of the remarkable X-ray observations which Giacconi has described for us.

\section{Accretion Onto Isolated Compact Objects}

The simplest case of accretion is that discussed by Bondi (1952), Shvartsman (1971) and Shapiro (1973a) in which the gravitating object is at rest relative to a surrounding medium. A characteristic length which enters into the problem is the so-called 'accretion radius'

$$
r_{A} \simeq \frac{G M}{c_{\infty}^{2}} \simeq 4.8 \times 10^{13} \frac{M}{M_{\odot}}\left(\frac{T_{\infty}}{10^{4} \mathrm{~K}}\right)^{-1}\left(\frac{2}{1+X}\right) \mathrm{cm},
$$

* Present address: Institute of Astronomy, Madingley Road, Cambridge CB3 0HA. 
where $c_{\infty}$ and $T_{\infty}$ denote the sound speed and temperature in the medium at distances $\gg r_{A}$ from the gravitating object. $r_{A}$ is the distance at which the escape velocity is $\sim c_{\infty}$. It is assumed in (1) that the gas consists predominantly of hydrogen (with ambient proton density $n_{\infty} \mathrm{cm}^{-3}$ ) and that $X$ is the fractional ionization. If the gas can be treated as a fluid, then the general nature of the flow (where $v$ is the inward velocity) is

$$
\begin{aligned}
& r \gg r_{A}:\left\{\begin{array}{l}
n \simeq n_{\infty} \\
v \propto r^{-2}
\end{array}\right. \\
& r \simeq r_{A}: v \simeq c_{\infty} \\
& r \gg r_{A}: \begin{array}{l}
v \propto r^{-1 / 2} \\
n \propto r^{-3 / 2}
\end{array} \quad \text { (free fall) }
\end{aligned}
$$

The precise details of the flow depend on the effective equation of state for the gas (and thus on whether - for example - radiative cooling is important), but the above is essentially correct if the behaviour of the gas can be characterised by a ratio of specific heats $\gamma<\frac{5}{3}$. For $\gamma=\frac{5}{3}$, we have a special case for which the solution is not unique. This is because the flow is not supersonic for $r \ll r_{A}$ as it is when $\gamma<\frac{5}{3}$ (since in this case $T \propto r^{-1}$ so the internal and kinetic energies increase at the same rate), so the inner boundary condition is relevant. The accretion rate turns out to be approximately

$$
\dot{M} \simeq 2 \times 10^{10}\left(\frac{M}{M_{\odot}}\right)^{2}\left(\frac{T_{\infty}}{10^{4} \mathrm{~K}}\right)^{-3}\left(\frac{2}{1+X}\right)^{3 / 2} n_{\infty} \mathrm{gm} \mathrm{s}^{-1},
$$

the precise value being dependent on the equation of state.

The above results remain adequate if the object is moving at speed $V$ relative to the medium, provided that this motion is subsonic. If $V \gtrsim c_{\infty}, c_{\infty}$ should be replaced by $\left(c_{\infty}^{2}+V^{2}\right)^{1 / 2}$ in the formula for $\dot{M}$. In this latter case, the object accretes all the material within a cylindrical column of radius $G M / V^{2}$ so the accretion rate $\propto V^{-3}$. The flow pattern near the object itself - which is, of course, the region where most of the energy is liberated - may still be almost spherically symmetrical if angular momentum is unimportant. As was pointed out by Salpeter (1964), this process would gradually decelerate a fast-moving object.

\subsection{NEUTRON STAR}

Where the accreting object has a 'hard' surface, as does a neutron star, all the kinetic energy of infall must emerge in radiation. This radiation amounts to $\sim 10^{20} \mathrm{erg} \mathrm{gm}^{-1}$ for a neutron star. The stopping distance of protons impacting on the surface at speeds $\gtrsim c / 3$ is larger than the mean free path of outgoing photons. Thus in general the energy emerges as thermal radiation. When $n_{\infty} \simeq 1 \mathrm{~cm}^{-3}$ the expected temperatures are in the range $10^{5}-10^{6} \mathrm{~K}$ and the power output in soft X-rays $\sim 10^{30} \mathrm{erg} \mathrm{s}^{-1}$ (Ostriker et al., 1970). (As was emphasised by Shvartsman (1971), the appropriate value of $T_{\infty}$ to take in (2) is always $\gtrsim 10^{4} \mathrm{~K}$, because photoionization would be important 
out to radii $\gg r_{A}$. The problem must therefore be treated self-consistently, $T_{\infty}$ being itself a function of $n_{\infty}$ ). Even though $\mathrm{X}$-rays from an individual isolated neutron star in the interstellar medium would not be detectable unless its distance were $\lesssim 10 \mathrm{pc}$, the integrated $\mathrm{X}$-ray emission from neutron stars accreting interstellar gas may in fact make a significant contribution to the soft X-ray background, because there may be $\gtrsim 10^{9}$ defunct pulsars in the galactic disc. Accretion onto neutron stars is discussed further in Section 3 in connection with binary X-ray sources.

\subsection{Stellar-Mass Black HOles}

When the central object is a black hole, however, we will only observe the energy radiated by the material before it is swallowed by the hole. If the infalling matter has no angular momentum, this radiated energy essentially derives from the ' $p \mathrm{~d} V$ work' done in compressing the gas. If the cooling time is long compared with the characteristic infall timescale, then Bondi's $\gamma=\frac{5}{3}$ solution is applicable, and only a small fraction of the energy will be radiated. For given conditions at infinity, this situation will prevail if the mass is below a certain critical value. Taking only bremsstrahlung and line cooling into account, this limit is given by

$$
\frac{M}{M_{\odot}} \lesssim 10^{4}\left(\frac{T_{\infty}}{10^{4}}\right)^{1 / 2} n_{\infty}^{-1} .
$$

This is well satisfied for a stellar-mass black hole in the interstellar medium. Material accreted by such an object would therefore heat up, rendering the environment of the black hole a very weak source of X-rays and $\gamma$-rays. Shvartsman (1971) and Shapiro (1973a) have discussed this situation in more detail. An additional effect which reduces the intensity of the emergent radiation, calculated fully by Shapiro, arises from the fact that the radiation is generated very close to the black hole by material almost freely falling inwards. Both the gravitational redshift and aberration are then important. In later unpublished work Shapiro shows that accretion of matter with zero net angular momentum onto a Kerr black hole differs only slightly from the Schwarzschild case. At the high temperatures attained close to the black hole $\left(T \simeq 10^{12} \mathrm{~K}\right)$ the particle mean free paths are so long that a fluid-dynamical treatment is not really self-consistent unless collective effects are operative. However the effects of an interstellar magnetic field have to be taken into account. If this field is dynamically important at $r_{A}$, it obviously affects the character of the flow. But even if it is initially negligible, the field lines will be stretched radially during the inflow (instead of being compressed isotropically). As a consequence, the magnetic energy density varies as $r^{-4}$, and is likely eventually to become dynamically important (bearing in mind that the accretion radius is typically $\sim 10^{8}$ times larger than the Schwarzschild radius). The effects of this field are: (a) it ensures fluid-like behaviour of the material, because the gyroradius becomes very small compared with the scale of the system; (b) synchrotron radiation by electrons becomes an important cooling process (indeed, Shvartsman (1971) and Shapiro (1973b) estimate that about $1 \%$ of the energy of the infall will be radiated, yielding 
a luminosity $3 \times 10^{30}\left(M / M_{\odot}\right)^{3 / 2} n_{\infty}^{1 / 2} \mathrm{erg} \mathrm{s}^{-1}$ with a flat spectrum that cuts off in the visible, or possibly the ultraviolet band;(c) the magnetic field may induce turbulent motions, leading to more efficient dissipation of the infall energy. Although this is the simplest accretion problem of physical interest, several interesting aspects of it still await a thorough discussion. For example, electron and proton temperatures would tend to become unequal (the electrons become relativistic at $r \simeq 10^{3} \mathrm{GM} / \mathrm{c}^{2}$, and so have an effective $\gamma$ of $\frac{4}{3}$ - and are also subject to radiative losses - whereas the protons, being always non-relativistic, have a $\gamma$ of $\frac{5}{3}$ ). It is unclear to what extent energy exchange between the species would equilibrate the temperature. (If this does happen, the effective $\gamma$ is $\frac{13}{9}$ if radiative losses are negligible).

When the cooling time is very short compared with the infall timescale, one might imagine that more radiation would be emitted. But this is not necessarily so, the reason being that once the gas has cooled down to a temperature such that $k T \ll G M / r$, the ' $p \mathrm{~d} V$ work' becomes a negligible fraction of the gravitational energy released. (The situation might, however, be changed if a small-scale magnetic field could maintain supersonic turbulent motions which dissipated via shockwaves; but this is another problem that has not yet been properly treated). The simple analysis suggests that the maximum radiative efficiency is attained in situations where the infall timescale and cooling timescale become comparable close to the Schwarzschild radius. Even then, mainly because of aberration and the gravitational redshift, the efficiency is only $\sim 1 \%$.

Spherically-symmetric accretion onto an isolated collapsed object in interstellar space is thus unlikely to lead to any readily detectable radiation. (If a source of this kind were to be detected, it might be characterised by irregular rapid variability). This conclusion holds whether the gravitational field around the object is given by the Schwarzschild metric or by the more general Kerr metric. If the black hole moves at speed $V$, material would be captured from within a cylinder of radius $r_{A}\left(c_{\infty} / V\right)^{1 / 2}$ (for $V \lesssim c_{\infty}$ ). Interstellar material would have an initial angular velocity of perhaps $\sim 10^{-7} \mathrm{rad} \mathrm{yr}^{-1}$ on the scale of a few parsecs, and it is easily confirmed that provided $V \gtrsim 1 \mathrm{~km} \mathrm{~s}^{-1}$, the associated angular momentum remains dynamically unimportant compared to the gravity of the black hole even when the material approaches the Schwarzschild radius. As Shvartsman (1971) and Novikov and Thorne (1973) point out, this conclusion might alter if there were interstellar turbulence on scales down to $\sim 10^{14} \mathrm{~cm}$. (Note that if $V=0$ we have a singular case when there is no strict steady state solution to the accretion problem, and angular momentum eventually becomes important for an arbitrarily small rotation velocity 'at infinity').

\subsection{SUPERMASSIVE BLACK HOLES}

Accretion onto isolated supermassive black holes was first discussed by Salpeter (1964). According to (2), the accretion rate goes as $M^{2}$, and this suggests that one might expect high luminosities from very massive collapsed objects even when the surrounding gas has the low density and high temperatures appropriate to the 
intergalactic medium. This topic was recently considered by Pringle et al. (1973), who showed that a galactic-mass collapsed object in a cluster of galaxies might have a luminosity of as much as $\sim 10^{46} \mathrm{erg} \mathrm{s}^{-1}$. The reason for expecting this high luminosity lies partly in the high mass, but also results from the likelihood that the infalling matter would have so much angular momentum that it would not fall radially inward but would instead form a disc, from which a radiative efficiency in the range $6-42 \%$ seems guaranteed (see Section 3). The spectral distribution of the radiation is uncertain, and depends on whether the magnetic fields become high enough for synchrotron emission to be the major cooling process.

Lynden-Bell (1969) and Lynden-Bell and Rees (1971) have considered the observable effects of black holes of masses $\sim 10^{8} M_{\odot}$ at the centres of galaxies. Such objects may be expected to form as the endpoint of the evolution of a quasar. Many of the observed forms of activity in galactic nuclei can be interpreted as effects of accretion of interstellar matter by a central black hole, but there are no strong reasons for favouring this model over the many others proposed to explain these phenomena.

\section{Compact Objects in Binary Systems: General Considerations}

I shall now attempt to review some of the theoretical implications of the remarkable new results which Giacconi has described in his paper (this volume, p. 147). Before doing so, however, it is perhaps worth recalling that the idea of X-ray sources being associated with close binary systems dates back to the earliest days of X-ray astronomy. It was Hayakawa and Matsouko (1964) and Zel'dovich and Guseynov (1965) who first made the suggestion that binary stars might be X-ray sources. After Sco X1 had been identified with an object reminiscent of an old nova, many other theorists proposed that X-ray sources involved transfer of matter from one star onto a compact companion (see Burbidge (1972) for an account of these developments). It is still unclear whether this is actually happening in Sco X1; but there now seems little doubt that it is the case for a major class of X-ray sources in the Galaxy.

What, then, can be said concerning the general nature of objects like Her X1, Cen X3, Cyg X1 and Cyg X3? The first general point is that the rapid variability suggests, though of course it does not prove, that a very small object - probably even smaller than a white dwarf - is involved. The gravitational potential well associated with such an object is very deep indeed, and accretion therefore provides an efficient energy source. If, as seems to be the case in the observed X-ray binaries, the compact object is in a close orbit - almost a grazing orbit - around another star, then a copious supply of material is available from the companion. Because the efficiencies are so high, the accretion rates need only be in the range $10^{16}-10^{18} \mathrm{gm} \mathrm{s}^{-1}\left(10^{-10}-10^{-8}\right.$ $\left.M_{\odot} \mathrm{yr}^{-1}\right)$ in order to produce the observed luminosities. These are modest compared to the inferred transfer rates in other binary systems, and could be supplied by a stellar wind even if the companion star does not overflow its Roche surface.

A second general point is that most of the gravitational energy is liberated deep in the potential well - at or near the surface of the compact object if it is a neutron 
star; within a few Schwarzschild radii if it is a black hole. Thus the effective dimensions of the source (assuming that the compact object is in the stellar mass range) are only $\sim 10^{6} \mathrm{~cm}$. If $10^{36}-10^{38} \mathrm{erg} \mathrm{s}^{-1}$ are radiated thermally from such a small region, a temperature high enough that the energy emerges predominantly in the X-ray band is therefore guaranteed.

In this report, I shall confine attention exclusively to the 'standard model' in which the X-ray source is regarded as being associated with either a neutron star or a black hole. Although, as Giacconi has described for us, the evidence favouring this model seems fairly compelling, the case is certainly not completely watertight; and one should bear in mind that some quite different interpretations for various aspects of these phenomena still remain tenable. I shall first outline the main features of the 'standard model' and then comment on how it may apply to some particular sources.

My reason for concentrating on this model is that it seems more plausible than any specific alternative so far proposed. Also this model has formed the basis for many detailed studies of these phenomena - indeed it will only be possible for me to give a sketch of most of this work, and some aspects of the problem will be left out entirely.

The X-rays binaries obviously involve all the problems connected with ordinary close binary systems - problems which are still ill-understood despite having been with us for many years - together with a whole range of new phenomena connected with the compact object. For convenience of exposition, it is convenient to split the subject into three parts: the mass transfer (relevant length scales $\sim 10^{11} \mathrm{~cm}$ ); the accretion disc (dimensions $\sim 10^{10} \mathrm{~cm}$ ); and the compact object itself, which is also the region where the $X$-rays are presumed to originate $\left(10^{6}-10^{8} \mathrm{~cm}\right)$. Unfortunately these three areas cannot be regarded as entirely disjoint, despite the very different length scales involved. For example, the $\mathrm{X}$-ray intensity and spectrum is probably determined by processes occurring close to the compact object, but it may nevertheless have an important influence on the flow of matter from the companion because of heating and radiation pressure effects.

\subsection{THE MASS TRANSFER $\left(\sim 10^{11} \mathrm{~cm}\right)$}

Much theoretical work has been based on the hypothesis that the companion star fills its Roche lobe, and that material flows across the Lagrangian point. It is important, however, to remember that these analyses are only strictly valid if the star corotates with the orbital period. This is probably quite a good assumption in these close systems, unless they were perturbed $\lesssim 10^{6}$ yr ago. Some calculations - for example, estimated limits on the masses of the X-ray sources - depend rather heavily on this postulate. It is also possible that the star does not fill its Roche lobe, but has a strong stellar wind. Gas streams may cause the optical emission lines observed in these systems (and also, incidentally, confuse attempts at radial velocity determinations). I shall say nothing about this topic, which is similar to that which arises in all close binary systems.

\subsection{THE ACCRETION DISC $\left(\lesssim 10^{10} \mathrm{~cm}\right)$}

By whatever process material is captured from the companion star, it is likely to have 
so much angular momentum that it cannot fall directly onto the compact object. The matter will instead dissipate its motions perpendicular to the plane of symmetry and form a differentially rotating disc, the rotational velocity at each point being approximately Keplerian, and then gradually spiral inwards as viscosity transports its angular momentum outwards. If the companion star is overflowing its Roche lobe, it is conventionally assumed that the matter joins the disc at the radius where its angular momentum relative to the compact object equals that of a Keplerian orbit. This argument suggests that the so-called 'hot spot' appears at a radius which is $\sim 20 \%$ that of the Roche lobe around the compact star. The structure of the outer part of the disc is not well understood. The disc must extend further out than the hot spot, because some of the material transferred from the companion star has to carry away the angular momentum - it cannot all be accreted by the compact object. A further complication is that the gravitational field of the companion star probably cannot be ignored in the outermost part of the disc, so the gas will not circulate in simple Keplerian orbits.

If the accreted matter is captured from a strong stellar wind, it will tend to have less net angular momentum; but the disc would still extend out to a radius $\gtrsim 10^{9} \mathrm{~cm}$ in general.

The structure of accretion discs has been discussed by many authors - for example Prendergast and Burbidge, 1968 (who considered a disc surrounding a white dwarf); Lynden-Bell, 1969; Pringle and Rees, 1972; Shakura and Sunyaev, 1973; Novikov and Thorne, 1973 - and the details will not be repeated here.

An obvious prerequisite for the existence of a disc (whose thickness must, by definition, be only a small fraction of its radius) is that radiative cooling should be efficient enough to remove most of the energy liberated by viscous friction, so that the internal energy is small compared with the gravitational binding energy, i.e.

$$
k T\left(1+\frac{p_{r}}{p_{g}}\right) \ll \frac{G M m_{p}}{r},
$$

where $p_{r} / p_{g}$ is the ratio of radiation pressure to gas pressure, and $m_{p}$ is the proton mass. For accretion flows with the parameters appropriate to X-ray sources the densities are high enough, and the timescales long enough, to ensure that (4) is almost certainly fulfilled. Also, the mass in the disc is gravitationally negligible compared to that of the central object.

If a steady state has been set up, the structure of the disc is governed by the following system of equations. First, the same mass flux $\dot{M}$ must flow across any radius $r$, so that

$$
\dot{M}=2 \pi r \int \varrho(r, z) v_{r}(z) \mathrm{d} z
$$

for all $r$, when $z$ is the coordinate perpendicular to the disc measured from the plane of symmetry.

A second, and somewhat less trivial, requirement is that the flux of angular momen- 
tum should be the same at all $r$. Angular momentum is transported inward by the accreted matter, but transported outward by the viscous stresses. The difference between these quantities represents the rate at which the central compact object is gaining angular momentum. Following Novikov and Thorne (1973) we assume that angular momentum is being accreted at a rate $\beta \dot{M}\left(G M r_{1}\right)^{1 / 2}$, where $r_{1}$ is the radius of the inner boundary of the disc. Since the specific angular momentum deposited on the compact object cannot exceed the Keplerian value at $r_{1}$, we have $\beta \leqslant 1$. One then finds that the heat dissipated per unit surface area of the disc at a radius $r>r_{1}$ is

$$
p(r)=\frac{3 \dot{M}}{4 \pi r^{2}} G \frac{M}{r}\left(1-\beta\left(\frac{r_{1}}{r}\right)^{1 / 2}\right) .
$$

It is important to note that $\beta$ is a second parameter which is not completely determined by $\dot{M}$ - one can imagine situations with the same $\dot{M}$ but different torques in the disc, and therefore different values of $p(r)$. When $r \gg r_{1}$, however, one finds, independently of $\beta$, that the energy radiated at radii $\geqslant r$ is 3 times larger than the energy lost by the accreted material while spiralling inward to that radius. The extra contribution arises because the viscous stresses transport energy outward as well as momentum. One might at first sight worry about the energy budget for the disc as a whole. However, when $\beta=1$ one finds that the total energy radiated, integrating over all $r \geqslant r_{1}$, is precisely equal to $M$ multiplied by the binding energy of Keplerian orbit of radius $r_{1}$; when $\beta=0$, the factor of 3 enhancement applies right in to $r=r_{1}$, but in this case the extra energy comes from viscous torques which apply a drag to the compact object.* (i.e. twice as much energy in this case is supplied by the central spinning object as comes from the infalling material itself.)

These deductions do not depend on the viscosity - if this is low, then the radial velocity $v_{r}$ is small, so the equilibrium value of $\varrho$ needed in order to give a given $\dot{M}$ must be high; and conversely. But to analyse the structure of the disc in any further detail one must know something about the viscosity, and this is the stumbling-block to further progress. Possible causes of viscosity include turbulence induced by the differential rotation, convective motions, or sheared magnetic fields. Pringle and Rees (1972) and Shakura and Sunyaev (1973) made specific simplifying assumptions about the viscosity, which enabled them to discuss the vertical structure of the disc (i.e. the balance between the pressure gradient perpendicular to the disc and the component of gravity in that direction), and the spectrum of the emergent radiation. However one has little confidence that one knows even the appropriate order of magnitude for the viscosity, and it therefore seems premature to discuss the spectrum of the disc in great detail. The dominant emission mechanism is probably thermal bremsstrahlung, though the spectrum may be appreciably distorted as a result of scatterings by the hot thermal electrons. All that can be said is that the effective tem-

\footnotetext{
* Note that the above analysis is strictly Newtonian. When one considers an accretion disc surrounding a black hole, then one finds that the energy radiated by the disc equals the energy lost by infalling matter when the black hole accretes a specific angular momentum appropriate to the circular orbits at the inner edge of the disc (see Novikov and Thorne (1973) for the details of the relativistic case).
} 
perature must be at least as high as the black body temperature needed to radiate a power $p(r)$.

\subsection{THE COMPACT OBJeCt AND THE X-RAY EMISSION $\left(10^{6}-10^{8} \mathrm{~cm}\right)$}

When the compact object is a black hole, the disc extends inward to the innermost stable circular orbit, the emission being concentrated within a few Schwarzschild radii. As discussed by Bardeen (this volume, p. 132) efficiencies of up to $40 \%$ are possible for accretion discs around Kerr black holes, the precise upper limit depending on how much of the emitted radiation is captured by the hole. Attempts to determine the expected radiation spectrum are impeded by our ignorance about the viscosity, which introduces far larger uncertainties than those corresponding to the difference between a Schwarzschild and extreme Kerr black hole. In general, the temperature decreases outwards and, even though the emission is thermal, the integrated spectrum may resemble a power law. Radiation from the outer parts of the disc would not be energetically significant unless, as discussed by Shakura and Sunyaev (1973) the disc is so thick in relation to its radius that X-rays from the inner regions are intercepted by the disc and reradiated at softer energies. Some further aspects of this model, as it may apply specifically to Cyg X1, are discussed later.

When the central object is a spinning, magnetised neutron star, a far more complex situation ensues, which has been discussed extensively by Pringle and Rees (1972), Davidson and Ostriker (1973), and Lamb et al. (1973). If the neutron star were unmagnetised, then the disc would extend inwards until the accreted material grazed the star's surface. If, however, the neutron star has a surface magnetic field of the same strength as is inferred for pulsars $\left(\sim 10^{12} \mathrm{G}\right)$ then the magnetic stresses will influence the dynamics out far beyond the surface of the star. We define the 'Alfven radius' to be that distance at which the magnetic stresses are comparable with the viscous stresses in the disc, i.e.

$$
\frac{\left(H\left(r_{\mathrm{A}}\right)\right)^{2}}{4 \pi} \simeq \varrho\left(r_{\mathrm{A}}\right) v_{r}\left(r_{\mathrm{A}}\right) v_{\theta}\left(r_{\mathrm{A}}\right) .
$$

The Alfvén radius depends on $\dot{M}$, but somewhat insensitively because $H^{2}$ depends on $r$ at least as steeply as $r^{-6}$; and for typical parameters $r_{\mathrm{A}}$ is $10-100$ times larger than $r_{*} \cdot r_{\mathrm{A}}$ is fortunately independent of the viscosity except insofar as this affects the scale height. This means that the radiation from the disc itself is relatively unimportant. Once matter penetrates within $r_{\mathrm{A}}$, it is constrained to follow the field lines. If the star has an oblique dipole field, the infalling matter will impact on the surface in the vicinity of the magnetic polar caps. The physics at $r \simeq r_{\mathrm{A}}$ is so complicated that one cannot really estimate which of the magnetic field lines can capture matter. These field lines will probably, however, be a subset of those which would have reached out to radii $\gtrsim r_{\mathrm{A}}$ in the absence of infalling plasma. This suggests that, when $r_{\mathrm{A}} \gg r_{*}$, the material will be channelled onto only a small fraction of the stellar surface.

The dominant radiation mechanisms would be bremsstrahlung or cyclotron radiation (including emission at the first few harmonics of the basic cyclotron frequency). 
Lamb et al. (1973) and Gnedin and Sunyaev (1973) have discussed the likely beam shape of the emergent radiation. If the dominant opacity were ordinary Thomson scattering, the radiation would tend to leak out of the sides of the accretion column, yielding a fan beam. If the magnetic field is so strong that the cyclotron frequency exceeds the radiation frequency under consideration, then electron scattering is inhibited for radiation propagating along the field direction, and also for radiation travelling across to the field which is polarised such that the electric wave vector is at right angles to the magnetic field. Realistic models can yield either pencil beams or fan beams, depending on the strength of the magnetic field and the polarization of the radiation. Modulation of this beam pattern each time the neutron star spins generates the X-ray pulse shape. The radiation would generally be expected to display a high degree of both linear and circular polarization.

Some other aspects of this scheme are discussed later in connection with particular sources.

\subsection{THE 'EDDINGTON LIMIT'}

An important role in these models is played by the so-called 'critical luminosity' or 'Eddington limit' at which radiation pressure balances gravity. If Thomson scattering provides the main opacity and the relevant material is fully ionized, then this luminosity is

$$
L_{\mathrm{edd}}=\frac{4 \pi G M m_{p}}{\sigma_{\mathrm{T}} c} \simeq 10^{38}\left(\frac{M}{M_{\odot}}\right) \mathrm{erg} \mathrm{s}^{-1},
$$

$\sigma_{\mathrm{T}}$ being the Thomson cross-section.

One might therefore expect that the accretion rate $\dot{M}$ could approach, but in no circumstances exceed, the value needed to yield this luminosity. Recently, Margon and Ostriker (1973) have in fact analysed the data on X-ray sources, and find that there does indeed seem to be a luminosity cut-off at around the expected value of $L_{\text {edd }}$ for $M \simeq M_{\odot}$, and that there is a class of sources whose luminosities cluster close to this value. Because this issue is an important one, it is perhaps worth pointing out that the 'Eddington limit' is physically significant only under relatively restrictive circumstances - circumstances which are not generally met by the kinds of X-ray source models usually considered.

As emphasized by Buff and McCray (1974) the luminosity of a source powered by accretion cannot even approach $L_{\text {edd }}$ if the effective cross section per electron is larger than $\sigma_{\mathrm{r}}$. This is quite likely to be the case for a source emitting soft X-rays, because the relevant opacity (unless all the ions are completely stripped) is then primarily due to photoionization, for which $\sigma \gg \sigma_{\mathrm{T}}$. If the value of $\dot{M}$ in binary X-ray sources is controlled by processes occurring near the surface of the companion star or the critical Roche surface, as in the 'self-excited wind' hypothesis (Basko and Sunyaev, 1973; Arons, 1973) then one might expect the luminosity to stabilise at a value well below $L_{\text {edd }}$.

There are, however, several types of situation where luminosities $\gg L_{\text {edd }}$ are possible 
especially under the extreme conditions prevailing near compact objects. Some of these are mentioned below:

(i) The effective opacity may be much less than that provided by Thomson scattering. In the context of X-ray sources this may, for instance, happen in the accretion column above the magnetic polar caps of neutron stars, where the scattering cross section is $\ll \sigma_{\mathrm{T}}$ for photons below the cyclotron frequency travelling along the magnetic field direction.

(ii) The Eddington limit can also be violated in any non-spherically-symmetric configuration. Consider again, for example, the accretion column near a magnetised neutron star. If the magnetic field does not modify the opacity and make the scattering highly anisotropic, then radiation will tend to escape from the sides of the column. This means that the radiation flux along the column, and therefore the pressure opposing gravity, is then less than it would be in an isotropic situation. (An analogous argument may also apply to accretion discs).

(iii) As has been pointed out by Lamb et al. (1973) there are conceivable circumstances when the luminosity may exceed $L_{\text {edd }}$ even when the appropriate cross section is $\sigma_{\mathrm{T}}$ and the accretion is isotropic. This is because $L>L_{\text {edd }}$ is merely the condition that infalling matter should be decelerated. But unless the total optical depth is sufficiently large, this does not guarantee that radiation pressure can halt the accretion. The infalling matter carries momentum across a sphere of radius $r$ at a rate $\dot{M} v(r)$, where $v(r)$ is of the order of the free fall speed. If its kinetic energy is converted into radiation at a radius $r_{\text {min }}$ the outward momentum flux, ignoring relativistic corrections, is $\sim(\dot{M} / 2 c)\left(v\left(r_{\min }\right)\right)^{2}$ (and less, of course, if the conversion efficiency is low). This means that the average photon must undergo more than $2 c / v\left(r_{\min }\right)$ scatterings if radiation pressure is to stem the accretion flow (unless the main contribution to the opacity comes from radii $r \gg r_{\text {min }}$ ).

(iv) The Eddington limit is of course irrelevant in an unsteady or explosive situation: it is, for instance, violated by factors $\sim 10^{5}$ in supernovae.

A luminosity exceeding $L_{\text {edd }}$ obviously entails a correspondingly higher accretion rate. If, however, the accretion has the high efficiency expected in X-ray sources, the observations seem to rule out a value of $\dot{M}$ of $\gtrsim 10^{-7} \mathrm{yr}^{-1}$ in all cases. If the companion star loses mass at a higher rate than this (as is likely at certain stages of stellar evolution, and as would seem required if the changes of orbital period in Cen X3 are attributable to mass loss from the companion star) then most of the material would presumably escape from the system.*

\section{Phenomena Observed in Particular Binary X-Ray Sources}

Her $X 1$ and Cen $X 3$ are clear candidates for systems when the $\mathrm{X}$-ray source is a

* The possibility has been raised (Zel'dovich et al., 1972; Ruffini and Wilson, 1973) that a neutron star may be able to accrete at a rate $\sim 10^{-3} M_{\odot} \mathrm{yr}^{-1}$ and get so hot that the energy escapes mainly as neutrinos, but the photon luminosity remains below $L_{\text {edd }}$. There seem severe doubts, however, about whether this situation could actually be set up by gradually increasing $M$, and whether it would be stable. 
neutron star (and it is gratifying that the mass of Her X1 seems to be within the allowable range for neutron stars). There are several observed phenomena which can be tentatively explained on the basis of this model. These systems are like pulsars in that rotation provides the 'clock'. However the X-ray power radiated derives not from rotational kinetic energy - which could maintain the observed X-ray luminosity of Her X1 for $\lesssim 10 \mathrm{yr}$ - but from accretion. (This, as Schwartsman has pointed out, suggests at least part of the reason why pulsars are not found in binary systems. An isolated spinning neutron star, surrounded only by diffuse interstellar gas, generates the electromagnetically driven relativistic wind which is believed to be a precondition for the coherent pulsed radio emission. When such an object is embedded in a denser environment, the pressure of the relativistic outflow cannot hold the external matter at bay, and we instead get accretion, manifesting itself in the emission of thermal $\mathrm{X}$-rays. One can estimate that Her X1 would have displayed pulsar-like behaviour only if its period were $\lesssim 0.1 \mathrm{~s}$ ).

\subsection{Changes in the pulse Period}

Since an accreting neutron star is not drawing on its rotational energy as its main power supply, it is not obvious whether its spin rate should slow down or speed up. An element of gas accreted by the star carries angular momentum corresponding to corotation at the Alfvén radius. This suggests that the spin rate would speed up on a timescale

$$
\frac{M}{\dot{M}}\left(\frac{r}{r_{\mathrm{A}}}\right)^{2} .
$$

Even though $M / \dot{M} \simeq 10^{8} \mathrm{yr}$, this 'lever-arm' effect certainly allows a speedup as rapid as that observed in Cen X3. There is, however, a possible opposing effect tending to brake the rotation: this is the viscous torque exerted by the accretion disc outside $r_{\mathrm{A}}$. These two effects can be of the same order of magnitude if

$$
\left(\frac{G M}{r_{\mathrm{A}}}\right)^{1 / 2} \simeq \Omega r_{\mathrm{A}}
$$

(and of course if $\left(2 G M / r_{A}\right)^{1 / 2}<\Omega r_{A}$ it would be energetically possible for material at the inner edge of the disc to be flung out of the system by magnetic forces, leading to a further braking effect). Davidson and Ostriker (1973) suggest that $\Omega$ tends asymptotically to a value such that the net torque on the neutron star is zero. This value of $\Omega$ depends on $r_{\mathrm{A}}$, which is itself a function of $\dot{M}$. Therefore, if there were fluctuations in the accretion rate, then $\Omega$ would tend to increase (decrease) as $\dot{M}$ increases (decreases). If Her X1 were close to this equilibrium state, and the fluctuations in $\dot{M}$ were small in amplitude, one could perhaps understand why $\Omega$ has been observed both to increase and to decrease, and why the timescale for these changes is slower than is the case for Cen X3.

The effects mentioned above are the dominant ones for causing changes in period. 
Other effects - for example, the spin-up due to the contraction of the star as it accretes mass - occur on the much slower timescale of $M / \dot{M}$.

\subsection{HER X1: THE LONG-TERM VARIABILITY}

Giacconi has summarized the X-ray data on the ' 35 day cycle' and I shall here briefly mention some of the numerous suggestions already made to explain this puzzling behaviour. In this connection, it is important to recall that optical observations impose an important constraint on such suggestions. It appears that the 1.7 day period light variations persist throughout the 35 day cycle with more or less the same amplitude (even though a 35 day periodicity may be discernable in some of the fine details of the light curve (Kurochkin, 1973; Boynton et al., 1973)). Since the thermal inertia of the relevant layers of the companion star is small, this implies that the heating mechanism operates throughout the $\sim 23$ days out of $\sim 35$ when UHURU detects no X-rays from Her X1.

\subsubsection{Modulations in Mass Transfer Rate}

One class of theory for the 35 day cycle involves supposing that the mass transfer is modulated with this period. It seems unlikely that this could be due to some pulsation of the companion star because the expected pulsation periods would be $\ll 35$ days. Another possibility (Pringle, 1973; Henriksen et al., 1973) is that the spin period of the companion differs by $\sim 5 \%$ from the orbital period. If the star displayed some departures from axisymmetry - a 'magnetic spot' associated with an especially vigorous wind for instance - then the transfer rate could vary with a synodic period of 35 days.

Conceivably some kind of feedback process may be operating. McCray (1973) has developed an ingenious model which utilises the fact that the X-ray luminosity is a significant fraction (perhaps $\sim 10 \%$ ) of $L_{\text {edd }}$. When the X-rays are 'on', the X-ray source behaves with respect to the surrounding gas as though it had a somewhat lower mass. The 'effective' Roche lobe around the companion star might then expand so that material no longer overflowed it. Mass transfer would then cease, and no material would be added to the disc. The disc would then drain away, and the X-ray emission would stop. Mass transfer would then begin again, the disc would be replenished, and so on. McCray speculates that some kind of limit cycle is set up. The time-scale of this cycle would be determined by the length of time taken for a typical element of gas to spiral inward to the central object. A period of the general order of 35 days would certainly not be unreasonable, but one cannot claim to 'predict' it, because of the wide uncertainty about the efficiency of viscosity in the disc.

A fully developed theory along these lines must also take account of a competing process which might cause positive feedback. This arises because the $\mathrm{X}$-rays, by heating the surface layers of the companion star, tend to raise the mass transfer rate by increasing the scale height in the atmosphere and/or by stimulating an enhanced stellar wind (Arons, 1973; Basko and Sunyaev, 1973; Alme, 1973). It has in fact been proposed (Lin, 1973) that the 35 day cycle could result from this type of positive 
feedback if the X-rays stimulate a mass transfer rate which 'overshoots' to such an extent that opacity effects around the compact object quench the X-rays. Plainly a proper theory of the 35 day cycle along the above lines must await a fuller understanding of how the $\mathrm{X}$-rays interact with the companion star, and also of the factors that determine the residence time of material in the accretion disc.

\subsubsection{Processes Occurring in the Accretion Disc}

Katz (1973) has suggested that the rim of the accretion disc may not lie in the orbital plane of the system. This might happen if the companion star possessed a component of spin angular momentum which was not aligned with the orbital angular momentum. In this situation, the rim of the disc would precess, and could obscure the X-rays for some fraction of each precession period. To obtain a precession period of 35 days, Katz has to assume that the disc extends outwards to a larger radius than is customarily supposed.

It is also conceivable that the disc might be subject to convective or other instabilities which might cause it to dump material periodically onto the central object.

\subsubsection{Modulation of Inflow from Alfvén Radius}

Pines et al. (1973) have developed a model according to which the neutron star undergoes free precession in such a way that the angle between the magnetic axis and the plane of the accretion disc varies periodically. When this angle is small, accretion along the 'magnetic funnel' can proceed; but when the magnetic axis points too far out of the plane of the disc accretion is suppressed, and material transferred from the companion accumulates in the disc outside the Alfvén radius. It is not clear how large the precession amplitude would have to be in order for such an 'accretion gate' to operate. However Pines et al. list some other reasons why the accretion flow near the Alfven surface could be sensitive to the orientation of the neutron star's rotation axis, so it is conceivable that a wobble through only a few degrees could suffice. On the basis of this model, Pines $e t$ al. have attempted to explain the other features of the 35 day cycle. The asymmetry between the sharp rise and the gradual fall in X-ray intensity during the 12 day 'on' period is readily explained. Matter accumulating during the 'off' period will be opaque to the X-rays until it has been photoionized. The fact that the switch-off occurs near orbital phases 0.25 or 0.75 is attributed to the higher density. of obscuring matter along the line joining the two stars, which makes it more likely that the first X-ray to be seen will escape perpendicular to this line. The hypothetical 'hot spot' where the gas stream merges with the disc may be thick enough to obscure the $\mathrm{X}$-rays at the phase of the orbit when it lies along our line of sight. The outer radius of the disc would decrease during the 'on' period, and the location of the hot spot would change (it is claimed) in such a way that the dip 'marches' in phase in the matter observed. The apparent tendency of the small amplitude $1.24 \mathrm{~s}$ optical pulsations (which probably come from gas with cooling time $\leqslant 1.24 \mathrm{~s}$ which is being heated by the X-rays) to occur at particular particular orbital phases can also be explained. 


\subsubsection{Precession of Pencil Beam}

Another idea involving precession of the neutron star (Brecher, 1972; Strittmatter et al., 1973) is that the X-rays remain 'on' for the whole 35 day cycle, but that they emerge in a pencil beam which sweeps through our line of sight only for 12 days out of 35. There are some geometrical difficulties associated with this idea. In particular, the broad and relatively smooth observed X-ray pulse profile tells us something about the shape of the beam, and it is hard to reconcile this with the sharp onset of the high state or with the apparent lack of any marked systematic changes in the pulse shape during the 'on' state. A very large wobble amplitude ( $\gtrsim 45 \mathrm{deg}$ ) would certainly seem required by this model.

At least in models (a) and (c), the continuous heating of the companion star can only be explained by invoking a steady heat source. One possibility (Avni et al., 1973) is that the neutron star emits a steady non-pulsed flux of soft X-rays, powered by the $\sim 8 \mathrm{MeV}$ per nucleon resulting from nuclear fusion of the accreted matter. This energy is liberated well below the neutron star surface, and emerges isotropically. But a serious problem arises with any model in which soft ( $\lesssim 0.5 \mathrm{keV}$ ) X-rays play the dominant role in the heating, because these photons (unlike harder X-rays) are absorbed predominantly above the photosphere. The associated energy input would then distort the temperature stratification, resulting in the formation of strong emission lines and suppression of the ordinary stellar absorption spectrum (Basko and Sunyaev, 1973; Strittmatter, 1973). It seems more likely that the star HZ Her is heated mainly by hard ( $~(10 \mathrm{keV}) \mathrm{X}$-rays, though the problem then is the inefficiency resulting from the high albedo (unless one considers photons of $\gtrsim 0.5 \mathrm{MeV}$ ). Heating by fast particles is another possibility. In models (b) and (d), one may suppose that $\mathrm{X}$-rays always hit the companion star even when they cannot propagate along our line of sight (though this requirement places further constraints on the geometry). A more attractive variant of (d) might be to postulate that the star is heated by hard $\mathrm{X}$-rays which are not so strongly beamed as those detected by UHURU. This is theoretically plausible because the circumstance which might most naturally cause a pencil beam - the reduced scattering cross section for photons travelling along the magnetic field direction - would not be so effective at high photon energies.

35 days is much too short a free precession period for a neutron star with a liquid core. However a neutron star with a solid core and the $1.24 \mathrm{~s}$ spin period appropriate to Her X1 could plausibly sustain a sufficient deviation from axisymmetry to yield a 35 day precession, and would then automatically be rigid enough to be able to wobble through a large angle. (Mechanisms for exciting this kind of wobble and for sustaining it against damping processes are discussed by Pines (1973).)

In assessing the various models for the 35 day cycle it is of course crucial to know just how regular a phenomenon it really is. It is also relevant that Cen X3 displays extended lows which are apparently not strictly periodic. Finally, some explanation is also required for the very long $(\sim 10 \mathrm{yr})$ timescale variability in Her X1 (Jones et al., 1973). If one were optimistic, one might therefore hope that two of the possibilities mentioned above might actually be relevant ! 


\subsection{Orbital Period of CEN X3}

The changes in the orbital period of Cen X3 have been attributed to a mass loss or mass transfer rate far higher than the minimum needed to provide the $\mathrm{X}$-ray power. However this large amount of gas, even if it were escaping from the system, would cause so much opacity that the X-ray source could not be observed. A more plausible possibility (Pringle, private communication) is that angular momentum is still being exchanged between the orbital motion and the spin of the companion star. This suggests that the event which formed the neutron star happened within the last $\sim 10^{4} \mathrm{yr}$, and that the timescale of the observed orbital period change corresponds to that required for tidal effects to establish synchronous rotation.

\subsection{CYG X1}

This is the prime candidate for being an X-ray source involving a black hole. One would expect the accretion disc around a black hole to be subject to various instabilities: thermal instabilities, magnetic instabilities (perhaps analogous to those which Parker has discussed in the context of the interstellar gas in our Galaxy), or perhaps instabilities resulting from irregularities in the mass transfer rate. These could give rise to irregular flickering on all time scales down to the orbital period associated with the most tightly bound stable circular orbits, but no regular period would be expected. Even if one had no evidence on its mass, one would therefore suspect Cyg X1 of being a disc around a black hole, and it is therefore gratifying that the evidence on its mass strongly supports this interpretation. Sunyaev (1973) proposed that attempts should be made to search for pulse trains due to regions of enhanced emissivity orbiting the hole. The typical orbital periods would be $\sim 0.5\left(M / M_{\odot}\right) \mathrm{ms}$ for a Schwarzschild black hole, but $\sim 8$ times faster if the black hole had a maximal Kerr metric whose angular momentum was aligned with the disc, but with the same mass. (Further interesting complications can occur if the black hole is obliquely oriented relative to the disc. Some of these have been mentioned by Bardeen in his review (this volume, p. 131)).

Further information would be derived if an X-ray spectral feature originating in the disc could be discovered and its profile measured, but this seems unlikely to be feasible before 1980. It is important to remember that black holes are a consequence of almost all 'viable' theories of gravity, and much further work is needed before one can diagnose whether the properties of a given black hole agree better with those expected on the basis of general relativity than with the predictions of a rival theory.

\subsection{Sco X1}

It is still unclear whether Sco X1 belongs to the same family as the other X-ray sources. Basko and Sunyaev have argued that Sco X1 could have a normal star as a binary companion with $L \lesssim L_{\odot}$. However, because the X-ray output is $\sim 10^{4} L_{\odot}$, the geometry and orientation of the system must be carefully specified in order to reduce the X-ray heating of the hypothetical companion to an acceptably low level.

The general spectrum of Sco Xl (in the infrared and optical bands as well as in 
$\mathrm{X}$-rays) is very well fitted by an accretion disc model (Pringle and Rees, 1972). Maybe Sco X1 could be a compact object surrounded by a massive disc, the disc being a remnant of a companion star destroyed by tidal forces (c.f. Faulkner, 1971).

\subsection{CYG X3}

The gradual and incomplete character of the X-ray eclipses suggests that in this system the eclipse is caused not by the surface of the companion star, but by scattering and absorption in a strong wind. The observed $2.2 \mu$ infrared variations imply that the relevant layer of the heated side of the companion star has a temperature $\gtrsim 10^{6} \mathrm{~K}$. This, however, is quite possible if one is seeing emission from the wind, which is heated to this temperature (Pringle, 1974).

Although Cyg X3 has a shorter period than the other X-ray binaries, the period is longer than that of systems such as DQ Herc. It may differ from such systems merely in having a neutron star (or black hole) as the compact component, instead of this being a white dwarf.

\section{Concluding Remarks}

Many important and interesting aspects of binary X-ray sources have not even been touched on in the foregoing review.

There is a whole complex of problems associated with the optical light curve of these systems. Heating of the companion star causes the side facing the compact object to be hotter and brighter than the eclipsed side. A full understanding of this effect involves detailed computations (along the lines of those already done by Arons (1973) and Basko and Sunyaev (1973)) of the structure of a stellar atmosphere irradiated by X-rays. A second quite different effect which leads to optical variations with half the orbital period arises from the distortion of the companion star by the compact object's gravitational field. Interpretation of actual light curves is complicated by further effects (emission by gas streams, radiation and absorption by the accretion disc itself, etc.) and one suspects that detailed model-building may prove somewhat fruitless unless some very clear-cut correlations between X-ray and optical variability are found. In Her X1, X-ray heating (or heating by some other radiation flux emanating from the compact object) is the dominant effect, and the effects of gravitational distortion are relatively minor; in Cyg X1, where the companion star is much more luminous relative to the X-ray source than is the case for Her X1, the heating augments the stellar luminosity by only $\sim 2 \%$. The occurrence of $X$-ray heating, however, sets a lower limit to the apparent brightness of the optical counterpart for any eclipsing X-ray source. If there were no interstellar absorption, any X-ray source with an intensity of $C$ UHURU counts which is observed to eclipse for a fraction $f$ of every period should have an optical apparent magnitude

$$
m \lesssim 15-2.5\left(\log \left(\frac{C}{10}\right)+2 \log (4 f)\right) .
$$


Thus any eclipsing source in the UHURU catalogue would be optically identifiable were it not for the often severe effects of interstellar extinction.

The radio properties of the $\mathrm{X}$-ray binaries are completely unexplained. However the fact that - even in the extreme case of Cyg X3 at the peak of its radio flare - the radio luminosity is a tiny fraction of the $X$-ray output, suggests that to concern ourselves with the details of the radio variability may be as premature as it would be to worry about solar flares before understanding the basic elements of stellar structure. Moreover, some binary systems containing two relatively normal stars have similar radio properties and this suggests that the radio behaviour is unlikely to be intimately connected to the compact object itself.

The existence of these close binary systems with compact components raises many astrophysical questions. How do they fit into the general scheme of binary star evolution? How do they evolve to their present state (and, in particular, how did they avoid disrupting during the catastrophe which formed the collapsed component)? Why, nevertheless are there only $\sim 30$ such systems in the Galaxy? What will be their eventual fate? - for example, what happens if a neutron star accretes so much material that it comes to exceed the limiting mass; or what happens when, later in its evolution, the companion star swells up and engulfs the compact object?

There is a real possibility of discovering an isolated massive black hole (as discussed in Section 2) by observing the effects of accretion. But X-ray binaries are the sole known instances of accretion onto relativistic systems. Far more detailed information can be expected from the next generation of $\mathrm{X}$-ray detectors (and from more refined optical observations) so there seems little doubt that these objects will remain at the focus of theoretical attention for several years to come, and will allow us to check the theory of black holes (and neutron stars) against observations in many key respects.

\section{References}

Alme, M.: 1973, paper presented at conference on Physics and Astrophysics of Compact Objects, Cambridge, England.

Arons, J.: 1973, Astrophys. J. 184, 539.

Avni, Y., Bahcall, J. N., Josse, P. C., Bahcall, N. A., Lamb, F. K., Pethick, C. J., and Pines, D.: 1973. Nature Phys. Sci. 246, 36.

Basko, M. M. and Sunyaev, R. A.: 1973, Astrophys. Sp. Sci. 23, 117.

Bondi, H.: 1952, Monthly Notices Roy. Astron. Soc. 112, 195.

Boynton, P. E., Canterna, R., Crosa, L., Deeter, J., and Gerend, D.: 1973, Astrophys. J. 186, 617.

Brecher, K.: 1972, Nature 239, 325.

Buff, J. and McCray, R. A.: 1973, Astrophys. J., in press.

Burbidge, G. R.: 1972, Comm. Astrophys. Space Phys. 4, 105.

Davidson, K. and Ostriker, J. P.: 1974,. Astrophys. J. 179, 585.

Faulkner, J.: 1971, Astrophys. J. Letters 170, L99.

Gnedin, Y. N. and Sunyaev, R. A.: 1973, Astron. Astrophys. 25, 233.

Hayakawa, S. and Matsouko, M.: 1964, Prog. Theor. Phys. Suppl. 30, 204.

Henriksen, R. N., Reinhardt, M., and Aschenbach, B.: 1973, Astron. Astrophys. 28, 47.

Jones, C. A., Forman, W., and Liller, W.: 1973, Bull. Am. Astron. Soc. 5, 32.

Katz, J. I.: 1973, Nature Phys. Sci. 246. 87.

Kurochkin, N. E.: 1973, Inform. Bull. Var. Stars, No. 55.

Lamb, F. K., Pethick, C. J. and Pines, D. : 1973, Astrophys. J. 184, 271. 
Lin, D. N. C.: 1973, Astron. Astrophys. 29, 109.

Lynden-Bell, D.: 1969, Nature 223, 690.

Lynden-Bell, D. and Rees, M. J.: 1971, Month.y Notices Roy. Astron. Soc. 152, 461.

Margon, B. and Ostriker, J. P.: 1973, Astrophys. J. 186, 91.

McCray, R. A.: 1973, Nature Phys. Sci. 243, 94.

Mestel, L.: 1954, Monthly Notices Roy. Astron. Soc. 114, 437.

Novikov, I. D. and Thorne, K. S.: 1973, in C. deWitt (ed.), Black Holes, Gordon \& Breach, p. 343.

Ostriker, J. P., Rees, M. J., and Silk, J. I.: 1970, Astrophys. Letters 6, 179.

Pines, D.: 1973, report presented at 16th Solvay Conference.

Pines, D., Lamb, F. K., and Pethick, C. J.: 1973, Proc. N. Y. Acad. Sci., in press.

Prendergast, K. H. and Burbidge, G. R.: 1968, Astrophys. J. Letters 151, L83.

Pringle, J. E.: 1973, Nature Phys. Sci. 243, 90.

Pringle, J. E. : 1974, Nature 247, 21.

Pringle, J. E. and Rees, M. J.: 1972, Astron. Astrophys. 21, 1.

Pringle, J. E., Rees, M. J., and Pacholczyk, A. G.: 1973, Astron. Astrophys., in press.

Ruffini, R. and Wilson, J.: 1973, Phys. Rev. Letters 31, 1362.

Salpeter, E. E.: 1964, Astrophys. J. 140, 796.

Shakura, N. I. and Sunyaev, R. A.: 1973, Astron. Astrophys. 24, 337.

Shapiro, S. L.: 1973a, Astrophys. J. 180, 531.

Shapiro, S. L.: 1973b, Astrophys. J., in press.

Shvartsman, V. F.: 1971, Soviet Astron. AJ 15, 377.

Strittmatter, P. A.: 1973, Astron. Astrophys. 185, 69.

Strittmatter, P. A., Scott, J., Whelan, J., Wickramasinghe, D. T., and Woolf, N. J.: 1973, Astron. Astrophys. 25, 275.

Sunyaev, R. A.: 1973, Soviet Astron. AJ 16, 941.

Zel'dovich, Ya. B. and Guseynov, O. K.: 1965, Astrophys. J. 144, 840.

Zel'dovich, Ya. B., Ivanova, L. N., and Nadezhim, D. K.: 1972, Soviet Astron. AJ 16, 209. 